Review article

\title{
Drug repositioning
}

\author{
Man Mohan Mehndiratta ${ }^{a, *}$, Swati Anil Wadhai ${ }^{b}$, Brij Kishor Tyagi $^{c}$, \\ Natasha Singh Gulati ${ }^{\mathrm{b}}$, Madhu Sinha ${ }^{\mathrm{b}}$ \\ a Department of Neurology, Janakpuri Super Specialty Hospital, Janakpuri, New Delhi 110058, India \\ ${ }^{\mathrm{b}}$ Department of Pathology, Janakpuri Super Specialty Hospital, Janakpuri, New Delhi 110058, India \\ 'Janakpuri Super Specialty Hospital, Janakpuri, New Delhi 110058, India
}

\section{A R T I C L E I N F O}

\section{Article history:}

Received 4 July 2016

Accepted 6 September 2016

Available online 10 October 2016

\section{Keywords:}

Drug repositioning

De-novo drug discovery

Pharmacotherapy

\begin{abstract}
A B S T R A C T
Rapid advances in pharmacotherapy and bioinformatics has led to the discovery and growing popularity of drug repositioning which includes re-investigating or recycling of existing drugs for new indications. There are innumerable advantages as well as challenges of drug repositioning. Since de-novo drug discovery takes plenty of time, effort and money, it has proved to a preferred alternative strategy for accelerated drug discovery. Moreover it is relatively inexpensive and carries minimal risk due to availability of previous pharmacological, safety and toxicology data. The strategies used are Known drug - new target/ Drug focus/Drug-centric, Known target- new indication/ Target focus/Target-centric and Disease focus/Disease-centric. Drug repositioning is a new breakthrough strategy to benefit patients by offering safer and effective treatment using shelved drugs.
\end{abstract}

(C) 2016 Published by Elsevier, a division of RELX India, Pvt. Ltd on behalf of Indian Epilepsy Society.
Drug repositioning is defined as the process of finding a new indication for an approved drug or abandoned pharmacotherapies. ${ }^{1}$ It identifies new indications of existing drugs and the application of the newly identified drugs to the treatment of diseases other than the drug's original indication. ${ }^{2}$ It involves analysis of drugs that have already been sanctioned for treatment of other diseases or whose targets have already been identified. Other synonyms are drug repurposing, drug rescue, redirecting, reprofiling, re-tasking, therapeutic switching and indication switching. ${ }^{3}$ It emerged primarily in the early 1990 s as an accidental discovery but lately due to development of new advents and tools the process has become more systematic. Repurposing of older drugs can fulfill vast unmet medical needs. It is an alternative to conventional de novo drug discovery and development. Repositioning is an accelerated approach for drug discovery because existing drugs have already passed pharmacokinetic and clinical analysis.

Presently drug repositioning project plays a pivotal role in the de novo drug discovery ventures of the pharmaceutical industry.

\footnotetext{
* Corresponding author at: President Asian and Oceanian Association of Neurology, Janakpuri Super Specialty Hospital, C-2/B, Janakpuri, New Delhi 110058 , India.

E-mail addresses: mmehndi@hotmail.com, mmehndi@gmail.com (M.M. Mehndiratta), swatiwadhai@gmail.com (S.A. Wadhai), drtyagispm@gmail.com (B.K. Tyagi),dr.ngulati@gmail.com (N.S. Gulati), madhushekharnath@rediffmail.com (M. Sinha).
}

The process of new drug development is tricky, time consuming and expensive. The pharmaceutical companies have not balanced output and the tremendous increase in research and development expenditure. ${ }^{4}$ This difference in productivity exists even though pharmaceutical companies have invested stupendous amounts in novel drug discovery technologies. ${ }^{5}$ The pharmaceutical companies today are facing downhill productivity with increased research and development expenditure. The pharmaceutical industry faces multiple challenges like, higher rates of attrition, drug safety issues, high regulatory pressures, expiring patent protection, and competition from generic drugs. ${ }^{6}$ The pharmaceutical companies are considering drug repositioning for accelerated drug discovery as it carries minimal risk of failure and is relatively inexpensive. Moreover they are always looking for developing drugs products which are economical, and carry limited risk strategy along with protection of existing products from competition and extension of their patent protection time. ${ }^{7}$ Drug repositioning is a low risk, high reward strategy as compared to de novo drug discovery, which is high risk, high reward strategy. There are 2000 failed drugs sitting that have the potential to develop into successful repositioned drugs. The list of failed drugs is increasing at the rate of approximately 150-200 compounds per year. 8,9

There are innumerable advantages of drug repositioning. It helps to recoup the existing expenditure, saves time and money with better implementation of sources. The cost to re-launch repositioned drug is quite reasonable ( $\sim 8.4$ million USD), whereas 
cost to re-launch the new formulation is extremely expensive ( $\sim 41.3$ million USD). The development of new drug costs more than 2.6 billion USD. ${ }^{10}$ So to launch the repositioned drug successfully to the market is quite economical than that of new drug. In clinical trials, repositioned drugs compete with nonrepositioned drugs in terms of efficacy rather than safety. Repurposed drugs escape much of the developmental cost than the traditional drug discovery due to availability of earlier pharmacokinetic, bioavailability, safety and toxicology data. Approximately 1 in 10,000 new drug that enters the clinical research and trial process, genuinely makes it to the market and roughly $30 \%$ of the drugs researched in clinical trials fail to qualify due to inefficacy. ${ }^{9,11,12}$ Thus repositioning ensures significant time and capital saving.

De novo drug discovery, right from the target identification to its development and approval takes almost $10-17$ years whereas approval of an existing drug takes 3-12 years. In standard drug

List of successfully repositioned drugs ${ }^{8,9,11,15-17}$ :

\begin{tabular}{|c|c|c|}
\hline Drug & Original indication & New indication \\
\hline Amantadine & Influenza & Parkinsonism \\
\hline Amphotericin & Antifungal & Leishmaniasis \\
\hline Aspirin & Inflammation, pain & Anti-platelet, stroke \\
\hline Amitripyline & Anti-depressant & Neuropathic pain \\
\hline Azathioprine & Rheumatoid arthritis (RA) & Multiple sclerosis (MS), inflammatory bowel disease (IBD) and organ transplants \\
\hline Bupropion & Depression & Smoking cessation and weight-loss (combi-therapy) \\
\hline Bimatoprost & Glaucoma & Eyelash growth \\
\hline Bromocriptine & Parkinson's disease & Diabetes mellitus \\
\hline Bleomycin & Antibiotic & Cancer \\
\hline Bromocriptine & Parkinson's disease & Type II diabetes \\
\hline Buprenorphine & Pain & Drug treatment \\
\hline Colchicine & Gout & Recurrent pericarditis and familial mediterranean fever \\
\hline Clofazime & Tuberculosis & Leprosy \\
\hline Canakinumab & Rheumatoid arthritis & Muckle-Wells syndrome \\
\hline Cyclosporine & Organ transplant rejection & Psoriasis and rheumatoid arthritis \\
\hline Colesevelam & LDL-lowering & Type II diabetes \\
\hline Crizotinib & Clinical trials for anaplastic large-cell lymphoma & Non-small cell lung carcinoma (NSCLC) \\
\hline Cycloserine & Tuberculosis & CNS disorders \\
\hline Dimethyl fumarate & Psoriasis & Multiple sclerosis \\
\hline Dapoxetine & Antidepressant & Premature ejaculation \\
\hline Doxepin & Antidepressant & Atopic dermatitis \\
\hline Donepezil & Alzheimer's disease & Dementia \\
\hline Duloxetine & Depression & Stress, fibromyalgia, urinary incontinence and chronic musculoskeletal pain \\
\hline Etanercept & Rheumatoid arthritis & Plaque psoriasis \\
\hline Everolimus & Immunosuppressant & Pancreatic neuroendocrine tumors \\
\hline Eflornthine & Cancer & Hirsutism and sleeping sickness \\
\hline Fluoxetine & Antidepressant & PMDD (premenstrual dysphoric disorder) \\
\hline Finasteride & Hypertension & Benign prostatic hyperplasia and male pattern baldness \\
\hline Galantamine & Chronic fatigue syndrome & Alzheimer's disease \\
\hline Gabapentin & Epilepsy & Neuropathic pain \\
\hline Glycopyrronium & Anti-ulcer & Chronic obstructive pulmonary disease (COPD) \\
\hline Gemcitabine & Anti-viral & Various cancers \\
\hline Hydroxychloroquine & Malaria & Lupus and rheumatoid \\
\hline Histrelin & Prostate cancer & Precocious puberty \\
\hline Imatinib & CML & Gastrointestinal stromal tumors \\
\hline Ibuprofen & Inflammation, pain & Osteoarthritis (OA), rheumatoid arthritis (RA), headache and migraine \\
\hline Iproniazid & Tuberculosis & Antidepressant \\
\hline Imfliximab & Autoimmune diseases & Crohn's disease \\
\hline Lomitapide & Hypercholesterimia & HoFH (homozygous familial hypercholesterolemia) \\
\hline Methotrexate & Cancer & Psoriasis and RA \\
\hline Miltefosine & Cancer & Visceral leishmaniasis \\
\hline Minoxidil & Hypertension & Male pattern baldness \\
\hline Milnacipran & Anti-depressant & Fibromyalgia \\
\hline Naltrexone & Opioid/alcohol addiction & Weight-loss (combi-therapy) \\
\hline Nelfinavir & Acquired immunodeficiency syndrome (AIDS) & In clinical trials for multiple cancers \\
\hline Onabotulinumtocin & Facial spasm & Chronic migraine, cervical dystonia and facial cosmetics \\
\hline Paroxetine & Antidepressant & Menopausal hot flashes \\
\hline Paclitael & Various cancers & Stent re-stenosis prevention \\
\hline Plerixafor & AIDS/HIV & Lymphoma and multiple myeloma \\
\hline Pertuzumab & Various cancers & HER-2 + breast cancer \\
\hline Pregabalin & Anticonvulsant and neuropathic pain & Fibromyalgia \\
\hline Pramipexole & Parkinson's disease & Restless leg syndrome \\
\hline Propranolol & Hypertension & Tremors, angina and migraine prophylaxis \\
\hline Raloxifene & Osteoporosis & Breast cancer \\
\hline
\end{tabular}

discovery, target discovery takes 2-3 years, screening and designing chemicals with biological activity takes 6 months to 1 year, lead optimization takes 1-3 years, 1-2 years to confirm drug ADMET (absorption distribution, metabolism, excretion and toxicity) properties using animal models, 5-6 years in clinical trial to determine drug safety and efficacy and 1-2 years for licensing. But in case of repositioning, compound identification takes 1-1.5 years, compound acquisition takes $0-1.5$ years, preclinical development 0-1 year, clinical trials 1-6 years, and 1-2 years for licensing. ${ }^{1}$ Few studies have shown that approval of a repositioned drug can be achieved in only 4 years. ${ }^{13}$ Thus by reducing length of time for development and re-launch of repurposed drug we can provide accelerated treatment options to the patients. Success rates for repurposed drugs are higher as compared to de novo drug discovery. Success rate for repurposed drugs was approximately $30 \%$ in recent years and was recently approved by the US FDA. ${ }^{14}$ 
(Continued)

\begin{tabular}{|c|c|c|}
\hline Drug & Original indication & New indication \\
\hline Retinoic acid & Acne & Acute myeloid leukemia \\
\hline Ropininole & Parkinson's disease & Restless leg syndrome \\
\hline Rituximab & Various cancers & Rheumatoid arthritis \\
\hline Sildenafil & Angina & Erectile dysfunction and pulmonary arterial hypertension \\
\hline Sunitinib & GIST and renal cell carcinoma & Pancreatic neuroendocrine tumors \\
\hline Trastuzumab & HER2-positive breast cancer & HER2-positive metastatic gastric cancer \\
\hline Thalidomide & Morning sickness & Multiple myeloma and erythema nodosum leprosum \\
\hline Zidovudine & Cancer & HIV/AIDS \\
\hline
\end{tabular}

There are two approaches of drug repositioning ${ }^{14}$ :

(i) Known drug - new target/drug focus/drug-centric where drug is already approved for specific indication and helps to identify its role in different indications. In this method single drug interacts with multiple targets.

(ii) Known target - new indication/target focus/target-centric

It identifies relevance between known targets of compounds and a new disease, i.e. discovers new medical potential of a new compound.

(iii) Disease focus/disease-centric

In this method we employ the acceptable experimental data related to disease and knowledge about how drugs modify phenotypes related to disease.

The technologies applied in drug repositioning comprises of in vivo (cell/organ/tissue/animal) phenotype model screening and in vitro, high content screening (HTC), high-throughput screening (HTS), chemoinformatics, data base driven bioinformatics information with Network and Systems Biology. These methods are used in association with available information on known target profile, pharmacokinetics of drugs, biomarkers of disease and disease pathway which will be time-saving and can lead to rapid discovery of drugs.

In spite of the entire efforts, drug repositioning still faces plenty of challenges and not all cases of drug repositioning are fruitful. Such as to test a specific drug for a considerable number of diseases or a generous number of drugs for a particular disease, it is challenging and laborious to consolidate the required computational approaches because the available information of drugs and disease may vary. ${ }^{14}$ In other cases drug such as bevacizumab (kinase inhibitor) was found to be ineffective in phase III trial for gastric cancer although being repositioned in other cancers. ${ }^{18}$ Even Sunitinib failed in clinical trials for colorectal cancer, breast cancer, prostate cancer and NSCLC, but has been successfully repositioned for treatment of renal cell carcinomas, GISTs and pancreatic neuroendocrine tumors. ${ }^{19}$ So looking at the ineffectiveness of Sunitinib in some cancers, we have to develop a targeted approach for some cancers. Repositioned drugs can cause adverse effect even after successfully passing the clinical safety standards. For e.g., the combination of naltrexone and bupropion was previously approved for the treatment of opioid addiction and depression respectively. It was also found to regulate energy consumption and appetite in obesity. However, due to the cardiovascular side effects of this combination, the FDA rejected this combination in 2011. . $^{20,21}$

The most rewarding base of drug repositioning is that we can start with an old drug. It is a strategy that revives old and dead drugs. There is still scope of research as there are still plenty of undiscovered therapeutic indications of known drugs. This approach has provided new source of income, market potential advantage, return on investment potential and money saving advantage to numerous pharmaceutical companies. Drug repositioning helps to recover the existing investment, saves time and money with better utilization of sources. It is a remunerative approach, helps to lessen the burden of diseases and accelerates productivity of pharmaceutical companies.

\section{Drug repositioning in epilepsy}

Due to complex nature of epilepsy and multi-target approach of anti-epileptics, drug repositioning can prove advantageous in the field of anti-epileptic drugs therapeutic armamentaria. Various studies have demonstrated anti-convulsant effects of anti-arrythmic drugs (AADs). Among class I AADs, Propafenone enhanced anticonvulsant action of classical anti-epileptic drugs (AEDs) ${ }^{22}$ and reduced maximal electroshock induced seizures in rats. ${ }^{23}$ Lidocaine (also an anesthetic agent) demonstrated anti-epileptic activity in patients with chronically unstable generalized epilepsy. ${ }^{24}$ Mexiletine was found to be effective in the treatment of symptomatic partial epilepsy and Lennox-Gastaut syndrome $e^{25-27}$ and decreased pentylenetetrazole-induced convulsions, and sound induced convulsions in DBA/2 mice. ${ }^{28}$ In class II AADs, Propranolol decreased seizures in patients with chronically unstable generalized epilepsy $^{29}$ and it demonstrated anticonvulsant action in models for generalized tonic-clonic and complex partial seizures, but not for myoclonic convulsions, ${ }^{30}$ Timolol reversed the epileptiform activity of pentylenetetrazol (PTZ) in the mouse brain. ${ }^{31}$ In class III AADs, Amiodarone inhibited PTZ - and caffeine-induced convulsions in mice. ${ }^{32}$ In class IV AADs, Verapamil protected mice against PTZinduced seizures and inhibited epileptogenesis in amygdalakindled rats ${ }^{33,34}$ and was found to be effective in the treatment of recurrent status epilepticus in human. ${ }^{35-37}$

Repositioning is going to gain much more importance in future as it can act as filler for ailing drug companies which are facing patent problems. In future, it can provide treatment options for rare diseases whose existing treatment options are very limited. Repositioned drugs have the same market potential as that of new drugs in the market and can also get the good market returns on the investment. Repurposing drugs can effectively lower the costs related to new drug development. It will be valuable for the patients because treatment options will be reasonable as compared to new drug discovery costs, which may not receive reasonable return on investment. Moreover, it is also important to develop both, as novel drug discovery can prevent exhaustion of supply and continuous screening of repositioned drugs can ensure full potential usage.

\section{Conflicts of interest}

The authors have none to declare.

\section{References}

1. Ashburn TT, Thor KB. Drug repositioning: identifying and developing new uses for existing drugs. Nat Rev Drug Discov. 2004;3:673-683.

2. Shim JS, Liu JO. Recent advances in drug repositioning for the discovery of new anticancer drugs. Int J Biol Sci. 2014;10(7):654-663.

3. Langedijk J, Mantel-Teeuwisse AK, Slijkerman DS, Schutjens MH. Drug repositioning and repurposing: terminology and definitions in literature. Drug Discov Today. 2015;20(8):1027-1034 
4. Landers P. Drug industry's big push into technology falls short. Wall Street J. 2004;(February):A1.

5. Usdin S. Industry development: pipeline or flatline? BioCentury. 2002;1:.

6. Sleigh SH, Barton CL. Repurposing strategies for therapeutics. Pharm Med. 2010;24:1519.

7. Smith RB. Repositioned drugs: integrating intellectual property and regulatory strategies. Drug Discov Today Ther Strateg. 2011;8:1317.

8. Corbett A, Williams G, Ballard C. Drug repositioning: an opportunity to develop novel treatments for Alzheimer's disease. Pharmaceuticals. 2013:6:1304-1321.

9. Persidis A. The benefits of drug repositioning. Drug Discov World Spring. 2011;9-12.

10. Cutting Edge Information. Pharmaceutical Product Relaunch: Preserving Market Share through Line Extension and New Market Entry Strategies. 2007, April http:// www.prnewswire.com/news-releases/drug-repurposing-the-most-affordablerelaunch-option-for-pharma-companies-58268372.html.

11. Padhy BM, Gupta YK. Drug repositioning: re-investigating existing drugs for new therapeutic indications. J Postgrad Med. 2011;57:153-160.

12. Kola I, Landis J. Can the pharmaceutical industry reduce attrition rates? Nat Rev Drug Discov. 2004;3:711-715.

13. Braun MM, Farag-El-Massah S, Xu K, Cote TR. Emergence of orphan drugs in the United States: a quantitative assessment of the first 25 years. Nat Rev Drug Discov. 2010;9(7):519-522.

14. Jin G, Wong S. Toward better drug repositioning: prioritizing and integrating existing methods into efficient pipelines. Drug Discov Today. 2014;19(5):637-644.

15. Gupta S, Sung B, Prasad S, Webb L, Aggarwal B. Cancer drug discovery by repurposing: teaching new tricks to old dogs. Trends Pharmacol Sci. 2013;34(9): 508-517.

16. Li YY, Jones SJ. Drug repositioning for personalized medicine. Genome Med. 2012;4(3):27.

17. Naylor S, Schonfeld JM. Therapeutic drug repurposing, repositioning and rescue. Part I. Overview. Drug Discov World Winter Ed. 2015;54-62.

18. Kang H, Kauh JS. Chemotherapy in the treatment of metastatic gastric cancer: is there a global standard? Curr Treat Options Oncol. 2011;12:96-106.

19. Blumenthal GM, Cortazar P, Zhang JJ, et al. FDA approval summary: sunitinib for the treatment of progressive well-differentiated locally advanced or metastatic pancreatic neuroendocrine tumors. Oncologist. 2012;17(8):1108-1113.

20. Plodkowski RA, Nguyen Q, Sundaram U, Nguyen L, Chau DL, St Jeor S. Bupropion and naltrexone: a review of their use individually and in combination for the treatment of obesity. Expert Opin Pharmacother. 2009;10(6):1069-1081.

21. Caveney E, Caveney BJ, Somaratne R, Turner JR, Gourgiotis L. Pharmaceutical interventions for obesity: a public health perspective. Diabetes Obes Metab. 2011;13(6):490-497.
22. Banach M, Piskorska B, Borowicz-Reutt KK. Propafenone enhances the anticonvulsant action of classical antiepileptic drugs in the mouse maximal electroshock model. Pharmacol Rep. 2016;68(June (3)):555-560.

23. von Philipsborn G, Gries J, Hofmann HP, et al. Pharmacological studies on propafenone and its main metabolite 5-hydroxypropafenone. Arzneimittelforschung. 1984;34:1489-1497.

24. Mori K, Ito H, Toda Y, et al. Successful management of intractable epilepsy with lidocaine tapes and continuous subcutaneous lidocaine infusion. Epilepsia. 2004;45:1287-1290.

25. Enoki H, Hata H, Ohmori I, Maniwa S, Ohta H, Kobayashi K. Clinical applications and the effect of mexiletine on refractory epilepsies (Japanese). No To Hattatsu. 2000;32:29-34.

26. Miyamoto A, Takahashi S, Oki J. A successful treatment with intravenous lidocaine followed by oral mexiletine in a patient with Lennox-Gastaut syndrome (Japanese). No To Hattatsu. 1999;31:459-464.

27. Nakazawa M, Okumura A, Niijima S, et al. Oral mexiletine for lidocaine-responsive neonatal epilepsy. Brain Dev. 2013;35:667-669.

28. Alexander GJ, Kopeloff LM, Alexander RB, Chatterjie N. Mexiletine: biphasic action on convulsive seizures in rodents. Neurobehav Toxicol Teratol. 1986;8:231-235.

29. de Oliveira GG, Borges MA. Propranolol action in chronically unstable generalized epilepsy. Am J Ther. 1994;1:38-41.

30. Borowicz KK, Banach M. Antiarrhythmic drugs and epilepsy. Pharmacol Rep. 2014;66:545-551.

31. Lathers CM, Stauffer AZ, Tumer N, Kraras CM, Goldman BD. Anticonvulsant and antiarrhythmic actions of the beta blocking agent timolol. Epilepsy Res. 1989;4:42-54.

32. Ozbakis-Dengiz G, Bakirci A. Anticonvulsant and hypnotic effects of amiodarone.J Zhejiang Univ Sci B. 2009;10:317-322.

33. Vezzani A, Wu HQ Stasi MA, Angelico P, Samanin R. Effect of various calcium channel blockers on three different models of limbic seizures in rats. Neuropharmacology. 1988;27:451-458.

34. Wurpel JN, Iyer SN. Calcium channel blockers verapamil and nimodipine inhibit kindling in adult and immature rats. Epilepsia. 1994;35:443-449.

35. Iannetti P, Spalice A, Parisi P. Calcium-channel blocker verapamil administration in prolonged and refractory status epilepticus. Epilepsia. 2005;46:967-969.

36. Schmitt FC, Dehnicke C, Merschhemke M, Meencke HJ. Verapamil attenuates the malignant treatment course in recurrent status epilepticus. Epilepsy Behav. 2010;17:565-568.

37. Summers MA, Moore JL, McAuley JW. Use of verapamil as a potential P-glycoprotein inhibitor in a patient with refractory epilepsy. Ann Pharmacother. 2004;38: $1631-1634$. 